\title{
El Trabajo Social y los Servicios Sociales en España: el precio del neoliberalismo
}

\author{
Blanca Gírela Rejón. Universidad de Granada \\ Recepción: 16 de febrero de 2017 | Aceptado: 6 de marzo de 2017 \\ Correspondencia: Blanca Gírela | Correo-e: bgirela@ugr.es
}

\author{
(iD 0000-0001-6855-3187 \\ Citar: Girela, B (2017). El Trabajo Social y los Servicios Sociales en España: el precio del \\ neoliberalismo. ReiDoCrea, 6, 95-104.
}

\begin{abstract}
Resumen: Hablar de Trabajo Social en España invita a una reflexión histórica sobre la trayectoria, origen y evolución del Trabajo Social en un país con fuertes influencias judeo-cristianas. Esta trayectoria no es unívoca y responde a cuestiones y acontecimientos políticos y culturales. La intención de este artículo es expresar el tipo de Trabajo Social que se desarrolla en España a expensas de modelos de Política Social neoliberales con todas sus amenazas y que erróneamente identifica al Trabajo Social, casi de forma exclusiva, con los Servicios Sociales y sus exiguas estructuras. Es necesario poner de relieve cuales han sido los sacrificios que desde las políticas sociales se han provocado en el Trabajo Social español, para que en su caso sean superados y resulten compatibles con un Trabajo Social liberador.
\end{abstract}

Palabras claves: Servicios Sociales | Neoliberalismo

\section{Social Work and Social Services in Spain: The Price of Neoliberalism}

\begin{abstract}
Speaking of Social Work in Spain invites a historical reflection on the trajectory, origin and evolution of Social Work in a country with strong Judeo-Christian influences. This trajectory is not univocal and responds to political and cultural issues and events. The aim of this article is to express the type of Social Work being carried out in Spain at the expense of neoliberal Social Policy models with all its threats and that erroneously identifies Social Work, almost exclusively, with Social Services and their meager structures. It is necessary to emphasize the sacrifices that have been made to Social Work in Spain as a result of social policies in order to improve them so they are compatible with a Liberating Social Work.
\end{abstract}

Keywords: Social Services | Neoliberalism

Identidad e Historia profesional

En cada época histórica, los trabajadores sociales tuvieron distintas oportunidades y en función de ellas buscaron diversas formas de promover el respeto a la dignidad de la persona humana, en cada época hubo entre los profesionales diversas formas concretas de adhesión a este postulado, -desde las más comprometidas a las más difusas- pero el valor del principio se mantuvo, se mantiene en la actualidad y se mantendrá en el futuro, como una gran opción ética de la profesión.

Nidia Aylwin (1999)

\section{Breve referencia histórica al Trabajo Social en Europa y su formación}

El Trabajo Social en Europa, desde una perspectiva histórica, está vinculado a una serie de acontecimientos claves, que por motivos de espacio y tiempo, vamos a abreviar. En primera instancia, hay que relacionar el origen del Trabajo Social en Europa con la Acción Social y el sentido que cobra esta. Como sabemos, la Acción Social en Europa ha discurrido de manera diferente en aquellos países en los que la hegemonía y la tradición católica eran evidentes. Tanto es así, que en esta cultura, la transferencia de responsabilidades sociales a la función pública, ha sido débil y poco definida, teniendo las instituciones religiosas un alto protagonismo en materia de 
atención social, que exime al Estado y administraciones públicas de muchas responsabilidades. La educación, la sanidad y la atención social han sido espacios ocupados por órdenes religiosas antes que por administraciones municipales. Contrario a esto, es el caso de países de tradición protestante ${ }^{1}$ en los que la responsabilidad pública y el reconocimiento de funciones, en materia de Acción Social, fueron clara y tempranamente reconocidas por el Estado.

Sin entrar en un relato muy detallado de la evolución histórica del Trabajo Social en España, pondremos nuestro punto de partida en la formación de los/as trabajadores/as sociales. Es evidente, que el Trabajo Social tiene que ver con el espacio profesional que se le delega y con la formación académica que se le atribuye. De este modo, se trata de ajustar perfiles académicos y profesionales a unas necesidades o requerimientos políticos. Dicho de otro modo, en España, como en otros muchos países, el Trabajo Social es y ha sido, funcional a las Políticas Sociales que se han desarrollado en determinados momentos, sirviendo este de mecanismo de control social. Y solo en los casos en los que el Trabajo Social se ha desvinculado de instituciones públicas, este ha podido ser más o menos radical y verdaderamente transformador.

Por su parte, la formación de los profesionales del Trabajo Social en Europa ha estado sometida a importantes cambios, sobre todo, desde que en el terreno universitario, se habla de Espacio Europeo de Educación Superior (EEES), que con la idea de favorecer la libre circulación de trabajadores entre países de la Unión, ha permitido la convergencia profesional y académica en la mayoría de las titulaciones. Pese a ello, los perfiles académicos y profesionales de cada país tienen identidad propia y responden a trayectorias concretas.

De todo ello se deduce, que en la mayoría de los países de la Europa occidental (con tradición democrática), la formación se centra en la Política Social, siendo la presencia curricular en Metodología del Trabajo Social, escasa y deficitaria. Algo que sorprende frente a países de Latinoamérica, que a pesar de haber estado sometidos a regímenes dictatoriales; "la metodología es exquisita, teniendo cabida en ella la sensibilización del pueblo y la intervención transformadora". (Rejón, 1984:66).

Por tanto, observamos que el afán del Trabajo Social por la Política Social y los Servicios de Bienestar ha hecho mella en la formación de los futuros trabajadores sociales, en detrimento de los discursos epistemológicos más punteros, que en la práctica no se aplicarán en esta forma de entender la profesión.

\section{La Unión Europea y el Trabajo Social}

En Europa, más que en ningún otro lugar, resulta del todo imposible, desvincular al Trabajo Social de la Política Social y los Servicios Sociales. Y esto, que en principio no es malo, resulta ciertamente preocupante, porque se diseñan Políticas Sociales y Servicios de Bienestar que tienen como finalidad la atención social y la emergencia social, a través del desarrollo de redes de servicios y prestaciones, que restan espacio a otro tipo de actuaciones profesionales y lo que es peor, en tiempos de crisis o de cambios políticos conservadores, estas redes disminuyen e incluso desaparecen, sumándonos los propios profesionales, a la precariedad laboral y social de nuestros/as usuarios/as. Baste como ejemplo el recién encuentro celebrado en Edimburgo en los primeros días de septiembre de 2015 y organizado por la Federación Internacional de

\footnotetext{
${ }^{1}$ La reforma protestante supone un punto de inflexión entre los modos de provisión y acción social en Europa con respecto a los países de tradición católica.
} 
Trabajadores Sociales, Región Europea (FITS, Europa), cuyo título es: "Servicios Sociales. Expo 2015". No hay nada de malo en celebrar un evento internacional para hablar, desde el Trabajo Social, de Servicios Sociales, el problema es que nuestro único campo profesional pueda ser este, porque en ese caso, en Europa nos estaremos convirtiendo en esquiroles de unas políticas sociales cada vez más insolidarias y restrictivas. $Y$ es que resulta, cuanto menos chocante, que los seminarios y redes de trabajo, tengan una visión tan patologizante de las realidades sociales y su forma de abordarlas, algo que denota la influencia de una Política Social de la Unión, caracterizada por un discurso neoliberal que se traduce en medidas y actuaciones, cada vez más residuales.

Es verdad, que no todas las políticas sociales en Europa son iguales, a pesar de las directrices comunitarias. Suecia, Dinamarca o Noruega, entre otros, nos inspira la idea de Bienestar Social, pero eso no es Trabajo Social. Tener redes de Servicios de Bienestar no se traduce en acción profesional. Muy por el contrario los/as trabajadores/as sociales se están convirtiendo en meros gestores de esas políticas sociales y aunque se lleven a cabo actividades profesionales dignísimas, el sistema constriñe nuestro ejercicio.

En el "Programa Mundial para el Trabajo Social y Desarrollo Social 2013" se hace un llamamiento a los trabajadores sociales "para fortalecer el reconocimiento de la importancia de las relaciones humanas y para trabajar en la sostenibilidad del medio ambiente y la comunidad" (Lawrence y Lyons, 2013: 373). Esto pone de manifiesto la preocupación, que desde distintos foros, se está expresando en relación a identificar Trabajo Social con Política Social. La proliferación, cada vez más significativa, del ejercicio libre de la profesión, pone de relieve esta evidencia. Sin embargo, cualquier equipo profesional que quiera funcionar al margen de las administraciones públicas, lo va a tener muy difícil, porque cuanto menos, ha de incorporarse a las redes de Servicios de Bienestar, con todas sus condiciones e irremisiblemente, ha de aspirar a concursos públicos a efectos de financiación y proyectos.

Es decir, no hay Trabajo Social en Europa al margen de las administraciones públicas e incluso, al margen de las organizaciones supranacionales, que serán las que marquen las directrices. También es cierto, que las directrices y disposiciones europeas han sido herramientas muy eficaces en materia de igualdad y protección social. No hay que desestimar los esfuerzos comunitarios en esta materia, pero estas decisiones son políticas y están sometidas a la filosofía dominante, cada vez mas incompatible con el pluralismo de Bienestar (Johnson, 1990).

Ya en su momento, Johnson advertía de la necesidad de redefinir el Bienestar Social como respuesta a la contraofensiva neoliberal impuesta, desde los años ochenta, por Thatcher y Reagan, tras la caída del muro de Berlín. Y esta redefinición era una buena oportunidad para los/as trabajadores/as sociales, en la medida en que teníamos cabida en diferentes esferas del Bienestar (desde el sector público, la iniciativa social o el voluntariado, la ayuda mutua y el sector mercantil) para hacer un Trabajo Social más independiente o al menos, autónomo.

\section{Las Políticas Sociales y el Trabajo Social. El caso Español}

El origen y evolución del Trabajo Social en España ha estado vinculado a la Iglesia católica y más tardíamente, al régimen dictatorial del general Franco y sus políticas desarrollistas y tecnocráticas. Desde instituciones religiosas se han dado, tradicionalmente, respuesta a las necesidades sociales en España. Por eso, no es extraño que en 1932 vea la luz la primera "escuela de asistencia social para la 
mujer". Se trata de una escuela católica, filial de otra belga. Esta escuela tenía como fin: "dotar de nociones y entrenamiento técnico a quienes decidieran intervenir en la obra de mejora de la sociedad, en la línea del resto de escuelas y corrientes europeas de profesionalizar la asistencia caritativa"(Sanz, 2001:11).

Posteriormente las Escuelas de Trabajo Social, dirigidas por la Iglesia o por el Ministerio de Cultura (a partir de los años sesenta), van a ser la plataforma de preparación para mujeres profesionales, volcadas en la acción social, que tendrán como cometido articular la asistencia social del régimen dictatorial franquista. "Esta feminización es reflejo de nuestro origen y trayectoria y conlleva, sin lugar a dudas, implicaciones para con los estudios, el estatus y situación dentro de la estructura universitaria y la producción científica" (Morales y Vieitez, 2015:298). Mujeres que, en muchos casos, "pusieron su atención en la idea de cambio social y aunque el cariz de la profesión era fuertemente católico, podía venir representado por altas dosis de humanismo y filantropía" (Girela, 2014: 1851). Estas escuelas pioneras, también hicieron de algún modo "transgresiones" y se vieron influenciadas en los años setenta por el movimiento de reconceptualización Latinoamericano que llegó de la mano de maestros como Kisnerman, Ander Egg y Aylwin, entre otros.

Con la instauración de la monarquía y la conquista de la democracia, se produce en España un desarrollo de la legislación social sin precedentes, que sitúa a los/as trabajadores/as sociales en el epicentro del Bienestar Social y el desarrollo del Sistema Público de Servicios Sociales. Es precisamente este hecho, el que hizo a los profesionales alejarse del discurso teórico de la reconceptualización, cambiando el trabajo social liberador y radical, por la gestión de servicios y prestaciones que venían amparados por nuevos derechos sociales. Este proceso tiene especial relevancia entre los años ochenta y noventa, cuando las escuelas de Trabajo Social, hoy facultades, empiezan a formar parte de las titulaciones universitarias con pleno derecho. Hasta entonces habían sido escuelas técnicas, con reconocimiento del Ministerio de Educación y Ciencia, pero sin el merecimiento de título universitario.

Son años de conquistas sociales lideradas, en gran medida, por los trabajadores/as sociales. Ahora bien, pasar de la asistencia social, a la gestión de los servicios sociales, ha supuesto para los/as trabajadores/as sociales "pagar un alto peaje" porque su labor se sobre-burocratiza y se pierde o se disipa la auténtica razón de ser del Trabajo Social. Durante estas décadas el Trabajo Social va a tener su expresión mayoritaria en la función pública, si bien la iniciativa social y el voluntariado complementarán estas actuaciones, en la práctica, la alta inspección de las administraciones: local, autonómica, nacional y europea estará presente. Por ello, hablar de Trabajo Social en España, más que nunca, estará vinculado a la trayectoria del Sistema Público de Servicios Sociales.

\section{De la Asistencia Social a los Servicios Sociales de Bienestar}

En España el Trabajo Social responde al modelo desarrollado de los países de la Europa meridional. Un modelo de Estado con fuertes sesgos familiaristas y en el que la Iglesia juega un papel determinante como subsidiaria del Estado en la provisión de recursos sociales. Este modelo ha determinado (a pesar de los cambios demográficos y los fenómenos de la globalización) un Trabajo Social afín a esta singularidad.

En los años sesenta y hasta los años ochenta del pasado siglo, en España se hablaba con más frecuencia de Asistencia Social y asistentes sociales para hacer referencia a la profesión y a la disciplina, como campo de estudio en instituciones académicas. Pero también era asistencia social la forma de acción social que desde estas 
instituciones se desempeñaba. Los profesionales estábamos a merced de un sistema social de protección, en distintas instancias, que era subsidiario a las posibilidades de la gente, no existiendo en estos años, derechos universales reconocidos y en donde se podría hablar de ciudadanos de primera y segunda categoría.

Con la asunción del Sistema Público de Servicios Sociales, en los últimos años de la década de los setenta y cuando se produce la democratización de los ayuntamientos, asistiremos a un incremento de la responsabilidad pública municipal en materia de acción social, que se traducirá en una importante presencia de profesionales, que desde instancias de gestión, dirección y técnicas van a protagonizar la implantación de programas sociales en términos de derechos sociales universales, que hasta entonces habían sido discrecionales.

Este panorama, que parece ideal, tiene sus aristas. Porque si anteriormente los las trabajadores/as sociales (con escasa presencia en la función pública y con escaso reconocimiento social) podían realizar acciones profesionales (también escasas) con la gente: dinamizando, creando conciencia, promoviendo las potencialidades y llevando a cabo una función profesional, a favor del cambio social, la nueva situación político administrativa lleva a los profesionales a ocupar puestos importantes en el diseño de las también nuevas políticas sociales y su ejecución. El prestigio y la presencia profesional van a ser evidentes. Sin embargo, lo que se nos va a pedir a los/as trabajadores/as sociales es que seamos instrumentos de esas novedosas políticas sociales, lo que va a suponer un alejamiento de las funciones propias para convertirnos en meros gestores de recursos.

En este nuevo panorama se pone el acento en los recursos sociales, en las prestaciones económicas y de servicios. Surge una nueva relación entre la ciudadanía y la función pública basada en el clientelismo. Nunca la población española estuvo más condicionada por los discursos políticos en materia de acción y promoción social. Pero en la práctica, esta promoción social es un arma de doble filo, porque realmente lo que acabamos haciendo los/as trabajadores/as sociales es dar cumplimiento a una serie de protocolos que determinarán quienes pueden acceder a determinados servicios o prestaciones y quienes no son merecedores de ellos. Nos convertimos en asignadores de recursos, en peritos de realidades sociales.

Si repasamos la literatura del Trabajo Social y los Servicios Sociales en España, podremos observar como los segundos han fagocitado al primero. La identificación del Trabajo Social con los Servicios Sociales ha sido tal, que parecía no poderse contemplar la acción profesional sin el Sistema Público de Servicios Sociales. Tanto es así, que el Consejo General de Colegios de Diplomados en Trabajo Social y Asistentes Sociales español, recoge en su página Web las áreas de «intervención ${ }^{2}$ » 0 de actuación de los mismos, refiriéndose a las siguientes: Sistema Público de Servicios Sociales, Dependencia y otros entre los que incluye: los Derechos Humanos, la Discapacidad y la Salud. Como puede observarse, se nos incluye en espacios públicos claramente imprescindibles, pero donde tenemos una función de contención social. Puede resultar escandaloso, pero desde nuestro punto de vista, se trata de mantener, en la mayoría de los casos, a la gente "en su lugar". Porque puede ser deliberado o no, pero áreas tan importantes como la Educación, la Vivienda y la Cooperación al Desarrollo tienen poca presencia de la profesión. De tal manera que en esta dinámica, lo que más y mejor hemos hecho los/as trabajadores/as sociales han sido los estudios de necesidades. $Y$ en este afán por detectar las necesidades de

\footnotetext{
2 Evitamos en este documento la palabra intervención porque consideramos que tiene connotaciones muy cuestionables. Se intervienen los bancos. Con las personas se actúa, se coopera y se participa.
} 
la gente, para poder actuar sobre ellas, lo que más no has preocupado es que se haga un «buen diagnóstico social». Pero al reflexionar sobre las necesidades de las personas, situamos a estas en un plano de vulnerabilidad y carencia; seres incompletos que algo errado han hecho para ser sujetos de ayuda.(Yazbek, 2012).

Queremos por tanto manifestar en este apartado, que el Trabajo Social en España, con una influencia Latinoamericana muy importante en lo teórico, ha estado transversalmente condicionado por la Política Social de los años ochenta y la puesta en marcha del Sistema de Servicios Sociales, que siendo importante para la atención social en España, ha condenado y reducido a profesionales del Trabajo Social a la gestión de recursos, desestimando, no por falta de ganas, sino de tiempo, cometidos que nos son propios y que a día de hoy son, en su caso, desatendidos u ocupados por educadores, personal sanitario, etc.

\section{La Unión Europea, los servicios sociales y el papel de los/as trabajadores/as sociales}

Los Servicios Sociales en España son junto a la Sanidad, la Educación, la Vivienda, la Protección del empleo y la Seguridad Social, uno de los seis Sistemas Públicos de Protección Social. No obstante, por su naturaleza histórica y al tratarse de atenciones sociales básicas, es el sistema más joven y menos desarrollado en el ámbito del Bienestar Social en España.

El ejercicio profesional se desarrolla, de manera muy significativa en este Sistema (Banda et al. 1999). Tanto es así, que supera el $70 \%$ de la ocupación profesional. En este sentido, se pueden ampliar datos en las páginas Webs de los Consejos de Colegios de Trabajadores Sociales a nivel autonómico y local. Pero lo realmente importante del Trabajo Social en España, es que se desarrolla en los Servicios Sociales, perdiendo independencia y autonomía y teniendo escasa presencia en las áreas de Educación, Salud, Seguridad Social, etc. Todo esto hace que al Trabajo Social se le identifique, generalmente, con relaciones de ayuda residuales: cuestión de pobreza y emergencia social. Pero en cualquier caso, los Servicios Sociales no son un Sistema de Protección Social tan asentado como el Sanitario o el Educativo y si tenemos en cuenta que nuestro ejercicio profesional se centra, de manera muy intensa, en el mismo, tendremos como resultado un Trabajo Social basado en la provisión de servicios y prestaciones más que en las tareas de promoción, concientización, movilización, etc.

Ahora bien, presentado este panorama, que no deja de ser deficitario y que aleja al Trabajo Social de sus verdaderos cometidos, hemos de admitir que existen infraestructuras y plataformas muy interesantes y eficaces. Sin embargo, el problema es que estas "buenas intenciones" son incompatibles con políticas neoliberales. Políticas que en tiempos de crisis, en vez de atender a la población con más medidas de Bienestar Social, compensando los estragos del capitalismo y sus ciclos, actúan recortando presupuestos y dejando a su suerte a los más desfavorecidos, al tiempo que se dan indultos fiscales para las grandes fortunas. Todo esto genera un contingente de usuarios/as (siempre el mismo) que fluctúa de unos servicios a otros, provocando dependencia institucional. Además estos Servicios Sociales también dependen de subvenciones y recursos aleatorios, tanto es así que: "La dependencia de los servicios sociales de subvenciones y de recursos externos, hecho que sucede tanto dentro como fuera de la administración pública, han condicionado el día a día en el desempeño de la función del trabajador social, lo que nos lleva a plantearnos un nuevo horizonte en el rol de la figura del profesional" (Navarro y Marín, 2012: 720). 
Y es que lo que ocurre en nuestro país es que, en el empeño de dar respuestas a las necesidades sociales insatisfechas, la gestión de recursos y prestaciones ocupa gran parte del rol profesional. Pero además, la precariedad de los Servicios, siempre sujetos a presupuestos exiguos, hace que en la práctica, nos dediquemos a "repartir miseria" y a generar una relación con la población usuaria en términos de dependencia institucional. En este sentido Pastor (2009: 101) es contundente cuando afirma que: "Los Servicios Sociales municipales deben facilitar el acceso y la participación de los ciudadanos no como usuarios o meros beneficiarios de actividades, sino en la elaboración, diseño, seguimiento y evaluación de programas, en la gestión de los Centros de Servicios Sociales, en definitiva, en pasar de una participación "interesada", de meros "espectadores" a una participación "sustantiva". Desde esta orientación participativa será posible acercar los discursos de los actores implicados para articular las respuestas necesarias a los complejos escenarios a los que se enfrentan los Servicios Sociales municipales".

Como puede observarse la participación en los Servicios Sociales debe dejar de ser la relacionada con la recepción de prestaciones, para favorecer la incorporación de la población usuaria en la toma de decisiones y diseño de programas sociales.

\section{Crisis económica y Trabajo Social en España}

La crisis económica que ha azotado a Europa y Estados Unidos en los últimos años, por más que algunos se empeñen en hablar de una crisis mundial, ha supuesto un varapalo a los Servicios Sociales en España y por tanto, al Trabajo Social dentro de estos. Porque en tiempos de crisis y de carencias, lo razonable sería (y así lo hacen los países nórdicos) intensificar la actividades y coberturas de los Sistemas Públicos de Bienestar Social. Muy por el contrario, en España se han llevado a cabo reformas sociales enfocadas a la reducción de presupuestos. El gasto social ha caído de forma alarmante, dejando a su suerte a la población más vulnerable y modificando las clases sociales, siendo las clases medias las más afectadas. Hablamos de desempleo, pobreza infantil, pobreza energética, desahucios, etc.

En este panorama, el Sistema Público de Servicios Sociales (espacio en donde, como ya se ha dicho ejercen su desempeño profesional un importante colectivo de trabajadores/as sociales) se ha visto fuertemente afectado. Incapaz de dar respuestas a las demandas de la ciudadanía, porque estas han subido, pero los presupuestos han bajado, los/as trabajadores/as sociales también han perdido, muchos/as de ellos/as, su empleo. Al suspenderse programas sociales, los despidos laborales han sido exagerados. La acción profesional se ha constreñido a la emergencia social y los bancos de alimentos han pasado a ser una prioridad de primera fila de manos de las iglesias y colectivos altruistas.

En este contexto las movilizaciones profesionales no se han hecho esperar y como defensa del Sistema Público de Servicios Sociales, ha surgido la llamada "marea naranja" que sin ser un movimiento de desobediencia civil (que sería, desde nuestro punto de vista, muy acertado) ha supuesto una plataforma contestaría al neoliberalismo con logros y actuaciones muy importantes. Esta sin duda, ha sido la oportunidad para que los profesionales nos planteemos redefinir nuestras funciones y la razón de ser de los Servicios Sociales. Las respuestas al respecto han dado como resultado plataformas de Defensa del Trabajo Social ${ }^{3}$ y una frenética actividad en las

\footnotetext{
${ }^{3}$ Las plataformas de defensa del Trabajo Social ha surgido en red, desde las distintas provincias y una primera a nivel nacional. Lo interesante de ellas es que son un importante foro de opinión, debate y reflexión para la profesión.
} 
redes sociales, que entendemos favorece al Trabajo Social que ha de salir reforzado de esta cruel situación.

\section{Desafíos de futuro y deconstrucción de un modelo de Trabajo Social clientelista}

Durante este relato hemos puesto de manifiesto nuestra preocupación por el Trabajo Social Europeo y en particular el Español, advirtiendo que las políticas sociales, llevadas a cabo desde los servicios sociales, han reducido las acciones profesionales, siendo estas, en gran medida, las de gestión de programas y prestaciones sociales. Hemos denunciado que el Trabajo Social ha sido fagocitado, de algún modo, por la Política Social y que los discursos teóricos no siempre han caminado de la mano de las prácticas de los/as trabajadores/as sociales. Sirva esta reflexión para evidenciar la dificultad que tenemos los/as trabajadores/as sociales para conjugar nuestros compromisos ético-morales con el ejercicio profesional: Tal fenómeno tiene un aspecto agradable, pero otro desagradable. El aspecto agradable es el compresible orgullo profesional de poseer una alta conciencia moral y una posición que permite la crítica de una realidad que suele ser insatisfactoria. El aspecto desagradable es la no menos comprensible angustia -e, incluso nihilismo- con la que los profesionales viven las realidades del ejercicio de la profesión. (Salcedo, 2001, 9-10)

Como puede apreciarse la preparación teórica y los principios éticos y operacionales del Trabajo Social español, chocan de frente con las directrices comunitarias y políticas, más preocupadas en la provisión de servicios y prestaciones, que por la promoción y participación social. Esto ha generado un tipo de ejercicio profesional interferido por el clientelismo político y social. De modo que a cambio de votos, las instituciones de servicios de bienestar (controladas por las administraciones públicas autonómicas y locales) han secuestrado las decisiones de las personas en la creencia de obtener beneficios sociales y prebendas con solo comprometer el voto. Un voto que en algunos sectores de población, es más cautivo que nunca. Todo esto nos lleva a plantearnos, desde el Trabajo Social, la necesidad de impulsar más y mejor la participación social. Deconstruir este clientelismo puede ser también la forma de redefinir y mejorar al Trabajo Social.

En este sentido, apostamos por la participación como arma de poder. Es esta la que puede hacer posible el desarrollo de capacidades y potencialidades de las personas y de esta modo "flexibilizar, desburocratizar y universalizar los servicios del Bienestar Social y contribuir así a una forma de democracia verdaderamente participativa y no meramente representativa".(Espadas, 2001:45)

El bipartidismo, por su parte, ha hecho un flaco favor a los Servicios de Bienestar y en general, los distintos sectores de población van a favorecer el poder del clientelismo social y político desde sus distintas expresiones: participando en comicios de forma condicionada y en el peor de los casos, absteniéndose de ello. La participación social no es solo una cuestión de votos, sino que exige estar presente en el diseño, planificación, ejecución y evaluación de los proyectos sociales y si esta participación no se crea, con una transferencia de poder a la ciudadanía y se le facilita a los profesionales del Trabajo Social su presencia en estos espacios, difícilmente podamos romper con el clientelismo orquestado por partidos políticos que de un lado u otro (derecha e izquierda) se ponen de acuerdo para mantener sus "feudos".

Sería muy importante que desde el Trabajo Social (desde las distintas plataformas profesionales) se llegara a un pacto con las administraciones públicas, que permitiera la verdadera participación social de la ciudadanía. No se trata de declaraciones de intenciones (esas ya las tenemos), sino de propiciar espacios y tiempo para la 
dinamización social. Profesionales ahogados con las exigencias de la gestión de servicios, difícilmente podrán llevar a cabo un Trabajo Social digno y real.

\section{Conclusiones}

1. La trayectoria, origen y evolución del Trabajo Social en España y Europa no es unívoca y responde a cuestiones y acontecimientos políticos y culturales. Esto ha hecho que la formación de los/as trabajadores/as sociales esté condicionada por estos acontecimientos.

2. El Trabajo Social de cada país tiene que ver con el espacio profesional que se le delega y con la formación académica que se le atribuye. De este modo, se trata de ajustar perfiles académicos y profesionales a unas necesidades 0 requerimientos políticos.

3. En la mayoría de los países de la Europa occidental (con tradición democrática) y desde una perspectiva histórica, la formación se centra en la Política Social, siendo la presencia curricular en Metodología del Trabajo Social, escasa y deficitaria.

4. En Europa (particularmente la meridional) resulta del todo imposible, desvincular al Trabajo Social de la Política Social y los Servicios Sociales. Y su finalidad será la atención y la emergencia social.

5. Las tendencias actuales en materia de Bienestar Social en Europa están relacionadas con el Neoliberalismo imperante con vocación de contención social.

6. No hay Trabajo Social en Europa al margen de las administraciones públicas e incluso, al margen de las organizaciones supranacionales, que serán las que marquen las directrices.

7. La feminización de la profesión y la tradición católica han definido, no solo al Trabajo Social meridional, sino también a las políticas sociales que se han implementado.

8. La asunción del Sistema Público de Servicios Sociales ha condicionado al Trabajo Social en estos espacios, favoreciendo el clientelismo social y la dependencia institucional en muchos casos. Se ha dado demasiada importancia a los recursos y prestaciones sociales en detrimento de las actuaciones profesionales vinculadas con la promoción, la sensibilización y la concientización.

9. El hecho de que más del $70 \%$ de los/as trabajadores/as sociales españoles/as desempeñen sus tareas en la función pública y en particular en los Servicios Sociales, nos induce a interpretar la atención social española en clave residual. La poca presencia de profesionales en el sistema educativo y sanitario, entre otros, es una evidencia de lo expuesto.

10. La crisis económica ha evidenciado la necesidad de redefinir nuestras funciones y se presenta como una oportunidad para generar un giro epistemológico que ponga en valor nuestras verdaderas funciones.

11. Por todo lo expresado, la participación social se hace imprescindible y se presenta como arma de poder. Es esta la que puede hacer posible el desarrollo de capacidades y potencialidades de las personas y reducir las prácticas burocráticas y el exceso de tecnocracia en nuestros sistemas. 


\section{Referencias}

Aylwin, N. (1999). Identidad e historia profesional. Revista Colombiana de Trabajo Social, 13, 7-23.

Banda, T. et al. (1999). Investigación sobre la ocupación profesional de los trabajadores sociales. Cuadernos de Trabajo Social, 12, 107-136.

Espadas, M. (2001). La participación ciudadana en el Sistema de Servicios Sociales en Escuela Universitaria de Trabajo Social de Linares. Nuevas perspectivas y líneas de investigación en torno al Trabajo Social, 39-61.

García, R. y Marín, A. (2012). Nuevo horizonte en el rol del profesional: Reinventar el Trabajo Social. En Actas del IX Congreso Estatal de Facultades de Trabajo Social de la Universidad de Jaén, 715-734.

Girela Rejón, B. (2014). Trabajo Social en España, trayectoria y vinculación con la política social y los servicios sociales" en Libro homenaje al profesor Rafael Barranco Vela. Vol. II, 1849-1866. Pamplona: Aranzadi.

Johnson, N. (1990) El Estado de Bienestar en Transición: La Teoría y la Práctica del Pluralismo de Bienestar. Madrid. Ministerio de Trabajo y Seguridad Social.

\section{Web-grafías}

http://ec.europa.eu/esf/home.jsp?langld=es

http://www.juntadeandalucia.es/organismos/igualdadypoliticassociales.htm

https://www.teaming.net/plataformadefensatrabajosocialespana?lang=es_ES
Lawrance, S., \& Lyons, K. (2013). Social Works and Social Services in Europe - a changing landscape. Cuadernos de Trabajo Social, 26(2), 371-383.

Morales, A. y Vieitez, S. (2015). Traslaciones de una profesión feminizada. De la Asistencia Social al Trabajo Social (280-302). En Saleti Cuesta, L. (coord..) Traslaciones de los estudios feministas. Málaga: Perséfone.

Pastor, E. (2009). El Trabajo Social en las políticas públicas de proximidad: Responsabilidad y Compromiso Social. Humanismo y Trabajo Social, 8, 85-109.

Rejón, M. (1984). La formación de Trabajadores Sociales en el extranjero. Revista de Servicios Sociales y Política Social, 61-66.

Salcedo, D. (2001). La participación social en los servicios públicos desde la perspectiva del Trabajo Social. Nuevas perspectivas y líneas de investigación en torno al Trabajo Social, 9-24.

Sanz, A. (2001). Acción social y trabajo social en España. Una revisión histórica. Acciones e Investigaciones Sociales, 5-42.

Yasbek, M. (2012). Pobreza no Brasil contemporâneo e formas de seu enfrentamento. Serviço Social, 110, 288-322.

\section{Revisión por pares abierta | Open Peer Review}

Dra. Aranzazu Gallego Molinero. Facultad de Trabajo Social. Universidad de Granada

0000-0001-6929-5554

Dra. Inmaculada Sancho Frías. Facultad de Trabajo Social. Universidad de Granada 